Commun.Fac.Sci.Univ.Ank.Series A 1

Volume 62, Number 1, Pages 21-32 (2013)

ISSN $1303-5991$

\title{
SOME CHARACTERIZATIONS OF TIMELIKE AND SPACELIKE CURVES WITH HARMONIC 1-TYPE DARBOUX INSTANTANEOUS ROTATION VECTOR IN THE MINKOWSKI 3-SPACE $E_{1}^{3}$
}

\author{
HÜSEYIN KOCAYIĞIT, MEHMET ÖNDER AND KADRI ARSLAN
}

\begin{abstract}
In this study, by using Laplacian and normal Laplacian operators, some characterizations on the Darboux instantaneous rotation vector field of timelike and spacelike curves are given in Minkowski 3-space $E_{1}^{3}$.
\end{abstract}

\section{Introduction}

In the local differential geometry, the characterizations of special curves are very important and fascinating problem. Especially, finding a relation to characterize the curves has an important role in the curve theory. The well-known of these special curves is constant slope curve or general helix which is defined by the property that the tangent vector of the curve makes a constant angle with a fixed direction. A necessary and sufficient condition that a curve to be a general helix in Euclidean 3 -space is that the ratio of curvature to torsion be constant [17]. Helix is one of the most fascinating curves in science and nature. This curve can be seen in many subjects of science such as nanosprings, carbon nanotubes, $\alpha$-helices, DNA double and collagen triple helix, lipid bilayers, bacterial flagella in Escherichia coli and Salmonella, aerial hyphae in actinomycetes, bacterial shape in spirochetes, horns, tendrils, vines, screws, springs, helical staircases and sea shells (helico-spiral structures) (see $[5,13,20])$. Furthermore, in the fields of computer aided design and computer graphics, helices can be used for the tool path description, the simulation of kinematics motion or the design of highways, etc. [21]. So, many mathematicians focused their studies on these special curves in different spaces such as Euclidean space and Minkowski space $[1,7,8,9,16,17]$.

Furthermore, in [14] Mağden has given a similar characterization for the helices in the Euclidean 4-space $E^{4}$ and in [12], Kocayiğit and Önder have obtained the corresponding characterizations of timelike helices in the Minkowski 4-space

Received by the editors Jan 02, 2012; Accepted: June 18, 2013.

2000 Mathematics Subject Classification. 14H50, 53B30, 53C50.

Key words and phrases. Darboux instantaneous rotation vector, circular helix, general helix. 
$E_{1}^{4}$. Furthermore, Kocayiğit has obtained the general differential equations which characterize the Frenet curves in Euclidean 3 -space $E^{3}$ and Minkowski 3 -space $E_{1}^{3}$ [11].

Moreover, Chen and Ishikawa classified biharmonic curves, the curves for which $\Delta H=0$ holds in semi-Euclidean space $E_{v}^{n}$, where $\Delta$ is Laplacian operator and $H$ is mean curvature vector filed of a Frenet curve [4]. Later, Kocayiğit has studied biharmonic curves and 1-type curves i.e., the curves for which $\Delta H=\lambda H$ holds, where $\lambda$ is constant, in Euclidean 3 -space $E^{3}$ and Minkowski 3 -space $E_{1}^{3}$. He has showed the relations between 1-type curves and circular helix and the relations between biharmonic curves and geodesics. He also studied the harmonic 1-type curves and weak biharmonic curves, i.e., the curves for which $\Delta^{\perp} H=\lambda H$ and $\Delta^{\perp} H=0$ hold along the curve, respectively, where $\Delta^{\perp}$ is the normal Laplacian operator [11]. Barros and Gray studied the curves in the Euclidean space with harmonic mean curvature vector [3]. Further, Kılıç and Arslan considered the curves in Euclidean space with 1-type mean curvature vector [10]. Then, Arslan, Aydın, Öztürk and Ugail have studied biminimal curves in Euclidean spaces [2].

In this paper, we obtain some characterizations on the Darboux vector $\vec{W}$ of a timelike or spacelike curve in Minkowski 3-space $E_{1}^{3}$ and find the equations characterizing the general helices. Furthermore, we give some characterizations of the curves for which $\Delta \vec{W}=\lambda \vec{W}, \Delta \vec{W}=0, \Delta^{\perp} \vec{W}^{\perp}=\lambda \vec{W}^{\perp}$ and $\Delta^{\perp} \vec{W}^{\perp}=0$ hold, where $\lambda$ is constant. According to these conditions, we give the characterizations of helices.

\section{Preliminaries.}

The Minkowski 3-space $E_{1}^{3}$ is the real vector space $\mathrm{R}^{3}$ provided with the standard flat metric given by

$$
g=-d x_{1}^{2}+d x_{2}^{2}+d x_{3}^{2},
$$

where $\left(x_{1}, x_{2}, x_{3}\right)$ is a rectangular coordinate system of $E_{1}^{3}$. An arbitrary vector $\vec{v}=\left(v_{1}, v_{2}, v_{3}\right)$ in $E_{1}^{3}$ can have one of three Lorentzian causal characters; it can be spacelike if $g(\vec{v}, \vec{v})>0$ or $\vec{v}=0$, timelike if $g(\vec{v}, \vec{v})<0$ and null (lightlike) if $g(\vec{v}, \vec{v})=0$ and $\vec{v} \neq 0$. Similarly, an arbitrary curve $\gamma(s): I \subset \mathrm{R} \rightarrow E_{1}^{3}$ is spacelike, timelike or null (lightlike), if all of its velocity vectors $\gamma^{\prime}(s)$ are spacelike, timelike or null (lightlike), respectively [15]. We say that a timelike vector is future pointing or past pointing if the first compound of the vector is positive or negative, respectively. Let $\vec{a}=\left(a_{1}, a_{2}, a_{3}\right)$ and $\vec{b}=\left(b_{1}, b_{2}, b_{3}\right)$ be two vectors in $E_{1}^{3}$. Then the vector product of $\vec{a}$ and $\vec{b}$ is given by

$$
\vec{a} \times \vec{b}=\left(a_{2} b_{3}-a_{3} b_{2}, a_{1} b_{3}-a_{3} b_{1}, a_{2} b_{1}-a_{1} b_{2}\right) .
$$

The Lorentzian sphere and hyperbolic sphere of radius $r$ and center 0 in $E_{1}^{3}$ are given by 
and

$$
S_{1}^{2}=\left\{\vec{x}=\left(x_{1}, x_{2}, x_{3}\right) \in E_{1}^{3}: g(\vec{x}, \vec{x})=r^{2}\right\}
$$

respectively [18].

$$
H_{0}^{2}=\left\{\vec{x}=\left(x_{1}, x_{2}, x_{3}\right) \in E_{1}^{3}: g(\vec{x}, \vec{x})=-r^{2}\right\},
$$

Denote by $\left\{\vec{V}_{1}, \vec{V}_{2}, \vec{V}_{3}\right\}$ the moving Frenet frame along the curve $\gamma(s): I \subset \mathrm{R} \rightarrow$ $E_{1}^{3}$. For an arbitrary curve $\gamma(s)$ in the space $E_{1}^{3}$, the following Frenet formulae are given:

Case 1: Let $\gamma(s)$ be a timelike curve. Then, the Frenet formulae are given as follows

$$
\begin{gathered}
{\left[\begin{array}{c}
\nabla_{\gamma^{\prime}} \vec{V}_{1} \\
\nabla_{\gamma^{\prime}} \vec{V}_{2} \\
\nabla_{\gamma^{\prime}} \vec{V}_{3}
\end{array}\right]=\left[\begin{array}{lll}
0 & \kappa & 0 \\
\kappa & 0 & \tau \\
0 & -\tau & 0
\end{array}\right]\left[\begin{array}{l}
\vec{V}_{1} \\
\vec{V}_{2} \\
\vec{V}_{3}
\end{array}\right],} \\
g\left(\vec{V}_{1}, \vec{V}_{1}\right)=-1, g\left(\vec{V}_{2}, \vec{V}_{2}\right)=g\left(\vec{V}_{3}, \vec{V}_{3}\right)=1 .
\end{gathered}
$$

(See [19]). From (2.1) the Darboux instantaneous rotation vector of the frame $\left\{\vec{V}_{1}, \vec{V}_{2}, \vec{V}_{3}\right\}$ is given by $\vec{W}=-\tau \vec{V}_{1}-\kappa \vec{V}_{3}$.

Case 2: Let $\gamma(s)$ be a spacelike curve. Then the Frenet formulae are given by

$$
\begin{gathered}
{\left[\begin{array}{c}
\nabla_{\gamma^{\prime}} \vec{V}_{1} \\
\nabla_{\gamma^{\prime}} \vec{V}_{2} \\
\nabla_{\gamma^{\prime}} \vec{V}_{3}
\end{array}\right]=\left[\begin{array}{lll}
0 & \kappa & 0 \\
-\varepsilon \kappa & 0 & \tau \\
0 & \tau & 0
\end{array}\right]\left[\begin{array}{l}
\vec{V}_{1} \\
\vec{V}_{2} \\
\vec{V}_{3}
\end{array}\right],} \\
g\left(\vec{V}_{1}, \vec{V}_{1}\right)=1, g\left(\vec{V}_{2}, \vec{V}_{2}\right)=\varepsilon, g\left(\vec{V}_{3}, \vec{V}_{3}\right)=-\varepsilon .
\end{gathered}
$$

(See [19]). For this case, the Darboux instantaneous rotation vector of the frame $\left\{\vec{V}_{1}, \vec{V}_{2}, \vec{V}_{3}\right\}$ is given by $\vec{W}=\varepsilon \tau \vec{V}_{1}-\varepsilon \kappa \vec{V}_{3}$.

In the formulae given by (2.1) and (2.2), $\kappa$ and $\tau$ are curvature and torsion of the curve $\gamma(s)$, respectively, and $\nabla$ is the Levi-Civita connection given by $\nabla_{\gamma^{\prime}}=\frac{d}{d s}$ where $s$ is the arc length parameter of the curve $\gamma$.

Using the Darboux vector, the Frenet formulae in (2.1) and (2.2) can be given as follows,

$$
\nabla_{\gamma^{\prime}} \vec{V}_{i}=\vec{W} \times \vec{V}_{i}, \quad(1 \leq i \leq 3)
$$

where $\times$ shows the vector product in Minkowski 3-space $E_{1}^{3}$.

A unit speed curve $\gamma: I \rightarrow E_{1}^{3}$ is a general helix, if the curvature $\kappa$ and the torsion $\tau$ aren't constants, but $\frac{\kappa}{\tau}(s)$ is constant along the curve. A curve $\gamma: I \rightarrow E_{1}^{3}$ is a circle, if the curvature $\kappa$ is a non-zero constant and the torsion $\tau$ is zero along the curve. We call a curve as a circular helix, i.e., a screw line or $W$-curve if both of $\kappa \neq 0$ and $\tau$ are constants. 
The Laplacian operator of $\gamma$ is defined by

$$
\Delta=-\nabla_{\gamma^{\prime}}^{2}=-\nabla_{\gamma^{\prime}} \nabla_{\gamma^{\prime}}
$$

and the normal connection of $\gamma$ is defined by

$$
\begin{aligned}
& \nabla_{\gamma^{\prime}}^{\perp}=\chi(\gamma(I)) \times \chi(\gamma(I))^{\perp} \rightarrow \chi(\gamma(I))^{\perp} \\
& \nabla_{\gamma^{\prime}}^{\perp} \vec{\xi}=\nabla_{\gamma^{\prime}} \vec{\xi}-g\left(\nabla_{\gamma^{\prime}} \vec{\xi}, \vec{V}_{1}\right) \vec{V}_{1}, \quad\left(\forall \vec{\xi} \in \chi(\gamma(I))^{\perp}\right)
\end{aligned}
$$

where $\nabla_{\gamma^{\prime}}^{\perp} \vec{\xi}$ is the normal component of $\nabla_{\gamma^{\prime}} \vec{\xi}$ or normal covariant derivative of $\vec{\xi}$ with respect to $\gamma^{\prime}, \chi(\gamma(I))=\operatorname{sp}\left\{\vec{V}_{1}(s)\right\}$ and $\chi(\gamma(I))^{\perp}=s p\left\{\vec{V}_{2}(s), \vec{V}_{3}(s)\right\}$ is the normal bundle of the curve $\gamma$. The normal Laplacian operator of $\gamma$ is defined by

$$
\Delta^{\perp}=-\nabla_{\gamma^{\prime}}^{\perp}(2)=-\nabla_{\gamma^{\prime}}^{\perp} \nabla_{\gamma^{\prime}}^{\perp}
$$

$($ see $[4,6])$.

\section{Characterizations of Timelike Curves with respect to Darboux Vector.}

In this section, we give the differential equation which characterizes the timelike curves in $E_{1}^{3}$ with respect to the Darboux vector $\vec{W}$.

Theorem 3.1. Let $\gamma$ be a unit speed timelike curve in $E_{1}^{3}$ with Frenet frame $\left\{\vec{V}_{1}, \vec{V}_{2}, \vec{V}_{3}\right\}$, curvature $\kappa$, torsion $\tau$ and Darboux vector $\vec{W}$. The differential equation characterizing $\gamma$ according to the Darboux vector $\vec{W}$ is given by

$$
\lambda_{3} \nabla_{\gamma^{\prime}}^{3} \vec{W}+\lambda_{2} \nabla_{\gamma^{\prime}} \vec{W}+\lambda_{1} \vec{W}=0
$$

where

$$
\begin{aligned}
& \lambda_{3}=f^{2} \\
& \lambda_{2}=\tau f\left(\tau f+\kappa^{\prime \prime}\right)-\kappa f\left(\kappa f-\kappa^{\prime \prime \prime}\right) \\
& \lambda_{1}=\kappa^{\prime} f\left(\kappa^{\prime \prime \prime}+\kappa f\right)-\tau^{\prime} f\left(\tau f+\kappa^{\prime \prime}\right)
\end{aligned}
$$

and $f=\tau^{\prime} \kappa-\kappa^{\prime} \tau$.

Proof. Let $\gamma$ be a unit speed curve with Frenet frame $\left\{\vec{V}_{1}, \vec{V}_{2}, \vec{V}_{3}\right\}$ and Darboux vector

$$
\vec{W}=-\tau \vec{V}_{1}-\kappa \vec{V}_{3}
$$

where $\kappa$ and $\tau$ are curvature and torsion of the curve, respectively. By differentiating $\vec{W}$ three times with respect to $s$, we find the followings,

$$
\nabla_{\gamma^{\prime}} \vec{W}=-\tau^{\prime} \vec{V}_{1}-\kappa^{\prime} \vec{V}_{3}
$$




$$
\begin{gathered}
\nabla_{\gamma^{\prime}}^{2} \vec{W}=-\tau^{\prime \prime} \vec{V}_{1}+\left(\kappa^{\prime} \tau-\kappa \tau^{\prime}\right) \vec{V}_{2}-\kappa^{\prime \prime} \vec{V}_{3}, \\
\nabla_{\gamma^{\prime}}^{3} \vec{W}=\left(-\tau^{\prime \prime \prime}+\kappa \kappa^{\prime 2} \tau^{\prime}\right) \vec{V}_{1}+\left(-\kappa \tau^{\prime \prime}+\left(\kappa^{\prime} \tau-\kappa \tau^{\prime}\right)+\kappa^{\prime \prime} \tau\right) \vec{V}_{2} \\
+\left(-\kappa^{\prime \prime \prime}-\kappa \tau \tau^{\prime}+\kappa^{\prime 2}\right) \vec{V}_{3} .
\end{gathered}
$$

From (3.2) and (3.3), we have

$$
\begin{aligned}
& \vec{V}_{1}=\left(\frac{\kappa}{\kappa^{\prime} \tau-\kappa \tau^{\prime}}\right) \nabla_{\gamma^{\prime}} \vec{W}-\left(\frac{\kappa^{\prime}}{\kappa^{\prime} \tau-\kappa \tau^{\prime}}\right) \vec{W}, \\
& \vec{V}_{3}=\left(\frac{\tau}{\kappa^{\prime} \tau-\kappa \tau^{\prime}}\right) \nabla_{\gamma^{\prime}} \vec{W}-\left(\frac{\tau^{\prime}}{\kappa^{\prime} \tau-\kappa \tau^{\prime}}\right) \vec{W} .
\end{aligned}
$$

By substituting (3.6) and (3.7) in (3.4), we get

$$
\vec{V}_{2}=\left(\frac{1}{\kappa^{\prime} \tau-\kappa \tau^{\prime}}\right) \nabla_{\gamma^{\prime}}^{2} \vec{W}+\left(\frac{\kappa^{\prime \prime} \tau-\kappa \tau^{\prime \prime}}{\left(\kappa^{\prime} \tau-\kappa \tau^{\prime 2}\right.}\right) \nabla_{\gamma^{\prime}} \vec{W}-\left(\frac{\kappa^{\prime} \tau^{\prime \prime}+\kappa^{\prime \prime} \tau^{\prime}}{\left(\kappa^{\prime} \tau-\kappa \tau^{\prime 2}\right.}\right) \vec{W}
$$

Now writing (3.6), (3.7) and (3.8) in (3.5) it follows,

$$
\begin{array}{r}
f^{2} \nabla_{\gamma^{\prime}}^{3} \vec{W}+f\left(g-f^{\prime}\right) \nabla_{\gamma^{\prime}}^{2} \vec{W}-\left[g\left(g-f^{\prime}\right)-\tau f\left(\tau f+\kappa^{\prime \prime}\right)+\kappa f\left(\kappa f-\kappa^{\prime \prime \prime}\right)\right] \nabla_{\gamma^{\prime}} \vec{W} \\
-\left[\left(f^{\prime}-g\right)\left(\kappa^{\prime} \tau^{\prime \prime}+\kappa^{\prime \prime} \tau^{\prime}\right)+\tau^{\prime} f\left(\tau f+\kappa^{\prime \prime}\right)-\kappa^{\prime} f\left(\kappa^{\prime \prime \prime}+\kappa f\right)\right] \vec{W}=0,
\end{array}
$$

where $f=\tau^{\prime} \kappa-\kappa^{\prime} \tau$ and $g=\kappa \tau^{\prime \prime}-\kappa^{\prime \prime} \tau$. Then we have $f^{\prime}=g$ and it gives

$$
\begin{aligned}
f^{2} \nabla_{\gamma^{\prime}}^{3} \vec{W} & +\left[\tau f\left(\tau f+\kappa^{\prime \prime}\right)-\kappa f\left(\kappa f-\kappa^{\prime \prime \prime}\right)\right] \nabla_{\gamma^{\prime}} \vec{W} \\
& +\left[\kappa^{\prime} f\left(\kappa^{\prime \prime \prime}+\kappa f\right)-\tau^{\prime} f\left(\tau f+\kappa^{\prime \prime}\right)\right] \vec{W}=0,
\end{aligned}
$$

By writing

$$
\begin{aligned}
& \lambda_{3}=f^{2}, \\
& \lambda_{2}=\tau f\left(\tau f+\kappa^{\prime \prime}\right)-\kappa f\left(\kappa f-\kappa^{\prime \prime \prime}\right), \\
& \lambda_{1}=\kappa^{\prime} f\left(\kappa^{\prime \prime \prime}+\kappa f\right)-\tau^{\prime} f\left(\tau f+\kappa^{\prime \prime}\right),
\end{aligned}
$$

from (3.9) we obtain (3.1).

Assume that $\gamma$ is not a planar curve. So, we can define a 2-dimensional subbundle, say $\vartheta$, of the normal bundle of $\gamma$ into $E_{1}^{3}$ as

$$
\vartheta=S p\left\{\vec{V}_{2}(s), \vec{V}_{3}(s)\right\},
$$

where $\gamma^{\prime}=\vec{V}_{1}(s), \quad \vec{V}_{2}(s)$ and $\vec{V}_{3}(s)$ are Frenet frame fields. Equations (2.5) and (2.6) also give how the normal connection $\nabla_{\gamma^{\prime}}^{\perp}$ of $\gamma$ into $E_{1}^{3}$ behaves on $\vartheta$

$$
\left\{\begin{array}{l}
\nabla_{\gamma^{\prime}}^{\perp} \vec{V}_{2}=\tau \vec{V}_{3} \\
\nabla_{\gamma^{\prime}}^{\perp} \vec{V}_{3}=-\tau \vec{V}_{2}
\end{array}\right.
$$


For the simplicity, we take $D_{\gamma^{\prime}}$ instead of $\nabla_{\gamma^{\prime}}^{\perp}$. Let us now denote the normal component of Darboux instantaneous rotation vector field $\vec{W}$ along $\gamma$ by

$$
\vec{W}^{\perp}=-\kappa \vec{V}_{3}
$$

where $\kappa$ is the curvature of $\gamma$. Then we give the followings.

Theorem 3.2. Let $\gamma$ be a unit speed timelike curve in Minkowski 3-space with Darboux vector $\vec{W}$. Then the differential equation characterizing $\gamma$ according to the normal component $\vec{W}^{\perp}$ is given by

$$
\lambda_{3} D_{\gamma^{\prime}}^{2} \vec{W}^{\perp}+\lambda_{2} D_{\gamma^{\prime}} \vec{W}^{\perp}+\lambda_{1} \vec{W}^{\perp}=0
$$

where

$$
\begin{aligned}
& \lambda_{3}=\kappa^{2} \tau, \\
& \lambda_{2}=\kappa\left(\kappa^{\prime} \tau+(\kappa \tau)^{\prime}\right), \\
& \lambda_{1}=\kappa^{\prime}\left(\kappa^{\prime} \tau+(\kappa \tau)^{\prime}\right)-\kappa \tau\left(\kappa^{\prime \prime 2}\right) .
\end{aligned}
$$

Proof. Let $\gamma$ be a unit speed timelike curve with Frenet frame $\left\{\vec{V}_{1}, \vec{V}_{2}, \vec{V}_{3}\right\}$ and the normal component

$$
\vec{W}^{\perp}=-\kappa \vec{V}_{3},
$$

where $\kappa$ and $\tau$ are curvature and torsion of the curve, respectively. By differentiating $\vec{W}^{\perp}$ two times with respect to $s$ we find the followings,

$$
\begin{gathered}
D_{\gamma^{\prime}} \vec{W}^{\perp}=\kappa \tau \vec{V}_{2}-\kappa^{\prime} \vec{V}_{3}, \\
D_{\gamma^{\prime}}^{2} \vec{W}^{\perp}=\left(2 \kappa^{\prime} \tau+\kappa \tau^{\prime}\right) \vec{V}_{2}+\left(-\kappa^{\prime \prime 2}\right) \vec{V}_{3} .
\end{gathered}
$$

From (3.14) and (3.15), we have

$$
\vec{V}_{2}=\frac{-1}{\kappa \tau}\left(\frac{\kappa^{\prime}}{\kappa} \vec{W}^{\perp}+D_{\gamma^{\prime}} \vec{W}^{\perp}\right) .
$$

By substituting (3.14) and (3.17) in (3.16) we get

$$
\begin{aligned}
\kappa^{2} \tau D_{\gamma^{\prime}}^{2} \vec{W}^{\perp} & +\kappa\left(\kappa^{\prime} \tau+(\kappa \tau)^{\prime}\right) D_{\gamma^{\prime}} \vec{W}^{\perp} \\
& +\left(\kappa^{\prime}\left(\kappa^{\prime} \tau+(\kappa \tau)^{\prime}\right)-\kappa \tau\left(\kappa^{\prime \prime 2}\right)\right) \vec{W}^{\perp}=0 .
\end{aligned}
$$

Writing

$$
\begin{aligned}
& \lambda_{3}=\kappa^{2} \tau, \\
& \lambda_{2}=\kappa\left(\kappa^{\prime} \tau+(\kappa \tau)^{\prime}\right), \\
& \lambda_{1}=\kappa^{\prime}\left(\kappa^{\prime} \tau+(\kappa \tau)^{\prime}\right)-\kappa \tau\left(\kappa^{\prime \prime 2}\right),
\end{aligned}
$$

we get the equality (3.13). 
Corollary 1. Let $\gamma$ be a unit speed timelike curve in $E_{1}^{3}$. If the curve $\gamma$ is a circular helix, then the differential equation characterizing the curve according to the normal Darboux vector $\vec{W}^{\perp}$ is given by

$$
D_{\gamma^{\prime}}^{2} \vec{W}^{\perp}+\tau^{2} \vec{W}^{\perp}=0
$$

and the normal component of Darboux vector of $\gamma$ is

$$
\vec{W}^{\perp}=c_{1} \cosh (\tau s)+c_{2} \sinh (\tau s),
$$

where $c_{1}, c_{2}$ are non-zero constants.

\section{Timelike Curves with Harmonic 1-type Darboux Vector.}

In this section we will give the characterizations of timelike curves with Harmonic 1-type Darboux vector in Minkowski 3-space $E_{1}^{3}$.

Definition 1. A regular timelike curve $\gamma$ in $E_{1}^{3}$ is said to have harmonic Darboux vector if

$$
\Delta \vec{W}=0,
$$

holds. Further, a regular timelike curve $\gamma$ in $E_{1}^{3}$ is said to have harmonic 1-type Darboux vector if

$$
\Delta \vec{W}=\lambda \vec{W}, \quad \lambda \in \mathrm{R},
$$

holds.

First we prove the following theorem.

Theorem 4.1. Let $\gamma$ be a unit speed timelike curve in $E_{1}^{3}$ with Darboux vector $\vec{W}$. Then, $\gamma$ has harmonic 1-type Darboux vector if and only if the curvature $\kappa$ and the torsion $\tau$ of the curve $\gamma$ satisfy the followings,

where $\lambda$ is constant.

$$
\left\{\begin{array}{l}
\tau^{\prime \prime}=-\lambda \tau, \\
\kappa \tau^{\prime}-\kappa^{\prime} \tau=0, \\
\kappa^{\prime \prime}=-\lambda \kappa,
\end{array}\right.
$$

Proof. Let $\gamma$ be a unit speed timelike curve in $E_{1}^{3}$ with Darboux vector $\vec{W}$ and let $\Delta$ be the Laplacian associated with $\nabla$. One can use (2.4) and (3.2) to compute

$$
\Delta \vec{W}=\tau^{\prime \prime} \vec{V}_{1}+\left(\kappa \tau^{\prime}-\kappa^{\prime} \tau\right) \vec{V}_{2}+\kappa^{\prime \prime} \vec{V}_{3} .
$$

We assume that the timelike curve $\gamma$ is of harmonic 1-type Darboux vector. Substituting (4.4) in (4.2), we have (4.3).

Conversely, if the equations (4.3) satisfy for the constant $\lambda$, then it is easy to show that $\gamma$ has harmonic 1-type Darboux vector.

Further, solving the system of differential equations in (4.3) we obtain the following corollary. 
Corollary 2. Let $\gamma$ be a unit speed timelike curve in $E_{1}^{3}$ with Darboux vector $\vec{W}$. Then, $\gamma$ has harmonic 1-type Darboux vector if and only if $\gamma$ is a general helix with curvature and torsion

$$
\begin{gathered}
\kappa=c \tau \\
\tau=c_{1} \cos (\sqrt{\lambda} s)+c_{2} \sin (\sqrt{\lambda} s)
\end{gathered}
$$

respectively, where $c, c_{1}, c_{2}$ are constants.

Corollary 3. Let $\gamma$ be a unit speed timelike curve in $E_{1}^{3}$ with Darboux vector $\vec{W}$. Then, $\gamma$ has harmonic Darboux vector if and only if $\gamma$ is a general helix with curvature and torsion

$$
\kappa(s)=c s, \quad \tau(s)=c_{1} s,
$$

where $c, c_{1}$ are constants.

Theorem 4.2. Let $\gamma$ be a unit speed timelike curve in $E_{1}^{3}$ with Darboux vector $\vec{W}$. Then,

$$
\Delta \vec{W}+\lambda \nabla_{\gamma^{\prime}} \vec{W}+\mu \vec{W}=0
$$

holds along the curve $\gamma$ for the constants $\lambda$ and $\mu$ if and only if $\gamma$ is a general timelike helix, with curvature and the torsion

$$
\begin{gathered}
\kappa=c \tau \\
\tau=c_{1} \exp \left(\frac{-\lambda+\sqrt{\lambda^{2}+4 \mu}}{2} s\right)+c_{2} \exp \left(\frac{\lambda-\sqrt{\lambda^{2}+4 \mu}}{2} s\right)
\end{gathered}
$$

where $c, c_{1}, c_{2}$ are constants.

Proof. Assume that (4.5) holds along the curve $\gamma$. Then from the equalities (3.2), (3.3) and (4.4) we have

$$
\left\{\begin{array}{l}
\tau^{\prime \prime}-\lambda \tau^{\prime}-\mu \tau=0 \\
\kappa \tau^{\prime}-\kappa^{\prime} \tau=0 \\
\kappa^{\prime \prime}-\lambda \kappa^{\prime}-\mu \kappa=0
\end{array}\right.
$$

The second equation of the system (4.6) gives that $\frac{\kappa}{\tau}$ is constant, i.e., $\gamma$ is a general helix. From the first and third equations, we get

$$
\tau=c_{1} \exp \left(\frac{-\lambda+\sqrt{\lambda^{2}+4 \mu}}{2} s\right)+c_{2} \exp \left(\frac{\lambda-\sqrt{\lambda^{2}+4 \mu}}{2} s\right)
$$

and

$$
\kappa=c \tau
$$


respectively, where $c, c_{1}, c_{2}$ are constants.

Conversely, if $\gamma$ is a general timelike helix with curvature $\kappa$ and torsion $\tau$ given by (4.8) and (4.7), respectively, it is easily seen that (4.5) holds.

\section{Timelike Curves with Harmonic 1-type Darboux Normal Component.}

In this section, we will give the characterizations of timelike curves with Harmonic 1-type Darboux normal component vector in Minkowski 3-space $E_{1}^{3}$.

Definition 2. A regular timelike curve $\gamma$ in $E_{1}^{3}$ is said to have harmonic Darboux normal component vector $\vec{W}^{\perp}$ if

$$
\Delta^{D} \vec{W}^{\perp}=0,
$$

holds. Further, a regular timelike curve $\gamma$ in $E_{1}^{3}$ is said to have harmonic 1-type Darboux vector if

$$
\Delta^{D} \vec{W}^{\perp}=\lambda \vec{W}^{\perp}, \quad \lambda \in \mathrm{R},
$$

holds, where $\Delta^{D}=-D_{\gamma^{\prime}} D_{\gamma^{\prime}}$.

Theorem 5.1. Let $\gamma$ be a unit speed timelike curve in $E_{1}^{3}$. Then, $\vec{W}^{\perp}$ is harmonic 1-type vector if and only if

$$
\left.\kappa^{\prime \prime 2}\right) \kappa=0, \quad 2 \kappa^{\prime} \tau+\tau^{\prime} \kappa=0 .
$$

Proof. Let $\gamma$ be a unit speed timelike curve in $E^{3}$ and let $\Delta^{D}=-D_{\gamma^{\prime}} D_{\gamma^{\prime}}$ be the Laplacian associated with $D$. From (3.16), we get

$$
\Delta^{D} \vec{W}^{\perp}=\left(2 \kappa^{\prime} \tau+\kappa \tau^{\prime}\right) \vec{V}_{2}+\left(\kappa \tau^{2}-\kappa^{\prime \prime}\right) \vec{V}_{3} .
$$

We assume that the normal component $\vec{W}^{\perp}$ of the Darboux vector field is of harmonic 1-type. Then substituting (5.4) in (5.2), we get (5.3).

Conversely, if the equations (5.3) satisfy then it is easily seen that the normal component $\vec{W}^{\perp}$ of the Darboux vector field is of harmonic 1-type.

Corollary 4. Let $\gamma$ be a unit speed timelike curve in $E_{1}^{3}$ with Darboux vector $\vec{W}$. If $\gamma$ is a circular timelike helix with torsion $\tau^{2}=\lambda$, then the normal component $\vec{W}^{\perp}$ of the Darboux vector field is of harmonic 1-type.

\section{Characterizations of the Spacelike Curves with respect to Darboux Vector.}

In this section, we give the characterizations of spacelike curves according to the Darboux vector. The proofs of this section can be obtained by the similar ways given in previous sections. 
Theorem 6.1. Let $\gamma$ be a unit speed spacelike curve in $E_{1}^{3}$ with Frenet frame $\left\{\vec{V}_{1}, \vec{V}_{2}, \vec{V}_{3}\right\}$, curvature $\kappa$, torsion $\tau$ and Darboux vector $\vec{W}$. The differential equation characterizing $\gamma$ according to the Darboux vector $\vec{W}$ is given by

where

$$
\lambda_{4} \nabla_{\gamma^{\prime}}^{3} \vec{W}+\lambda_{3} \nabla_{\gamma^{\prime}}^{2} \vec{W}+\lambda_{2} \nabla_{\gamma^{\prime}} \vec{W}+\lambda_{1} \vec{W}=0
$$

$$
\begin{aligned}
& \lambda_{4}=f^{2} \\
& \lambda_{3}=-2 f g \\
& \lambda_{2}=2 g^{2}-\tau f\left(\tau f-\kappa^{\prime \prime}\right)-\varepsilon \kappa f\left(\varepsilon \kappa^{\prime \prime \prime}-\kappa f\right) \\
& \lambda_{1}=2 g\left(\kappa^{\prime \prime} \tau^{\prime}-\kappa^{\prime} \tau^{\prime \prime}\right)+\tau^{\prime} f\left(\tau f-\kappa^{\prime \prime}\right)-\varepsilon \kappa^{\prime} f\left(\varepsilon \kappa^{\prime \prime \prime}-\kappa f\right),
\end{aligned}
$$

and $f=\kappa \tau^{\prime}-\kappa^{\prime} \tau, \quad g=\kappa \tau^{\prime \prime}-\kappa^{\prime \prime} \tau$.

Theorem 6.2. Let $\gamma$ be a unit speed spacelike curve in $E_{1}^{3}$. Then the differential equation characterizing $\gamma$ according to the normal component $\vec{W}^{\perp}$ is given by

where

$$
\lambda_{3} D_{\gamma^{\prime}}^{2} \vec{W}^{\perp}+\lambda_{2} D_{\gamma^{\prime}} \vec{W}^{\perp}+\lambda_{1} \vec{W}^{\perp}=0
$$

$$
\left\{\begin{array}{l}
\lambda_{3}=\kappa^{2} \tau \\
\lambda_{2}=-\kappa\left(\kappa^{\prime} \tau+(\kappa \tau)^{\prime}\right) \\
\lambda_{1}=-\varepsilon \kappa^{\prime}\left(\kappa^{\prime} \tau+(\kappa \tau)^{\prime}\right)-\kappa \tau\left(\kappa^{\prime \prime 2}\right) .
\end{array}\right.
$$

Corollary 5. Let $\gamma$ be a unit speed spacelike curve in $E_{1}^{3}$. If the curve $\gamma$ is a circular helix, then the differential equation characterizing the curve according to the normal component $\vec{W}^{\perp}$ is given by

$$
D_{\gamma^{\prime}}^{2} \vec{W}^{\perp}-\tau^{2} \vec{W}^{\perp}=0 .
$$

From the last differential equation, the normal component of Darboux vector of $\gamma$ is

$$
\vec{W}^{\perp}=c_{1} \exp (\tau s)+c_{2} \exp (-\tau s)
$$

where $c_{1}, c_{2}$ are non-zero constants.

\section{Spacelike Curves with Harmonic 1-type Darboux Vector and Harmonic 1-type Darboux Normal Component.}

Theorem 7.1. Let $\gamma$ be a unit speed spacelike curve in $E_{1}^{3}$ with Darboux vector $\vec{W}$. Then, $\gamma$ has harmonic 1-type Darboux vector if and only if the curvature $\kappa$ and the torsion $\tau$ of the curve $\gamma$ satisfy the followings,

$$
\tau^{\prime \prime}+\lambda \tau=0, \quad \kappa \tau^{\prime}-\kappa^{\prime} \tau=0, \quad \kappa^{\prime \prime}+\lambda \kappa=0,
$$

where $\lambda$ is constant. 
Corollary 6. Let $\gamma$ be a unit speed spacelike curve in $E_{1}^{3}$ with Darboux vector $\vec{W}$. Then, $\gamma$ has harmonic 1-type Darboux vector if and only if $\gamma$ is a general helix, with curvature and torsion

$$
\begin{gathered}
\kappa=c \tau \\
\tau=c_{1} \cos (\sqrt{\lambda} s)+c_{2} \sin (\sqrt{\lambda} s),
\end{gathered}
$$

respectively, where $c, c_{1}, c_{2}$ are constants.

Corollary 7. Let $\gamma$ be a unit speed spacelike curve in $E_{1}^{3}$ with Darboux vector $\vec{W}$. Then, $\gamma$ has harmonic Darboux vector if and only if $\gamma$ is a general helix with curvature and torsion

$$
\kappa(s)=c s, \quad \tau(s)=c_{1} s
$$

respectively, where $c, c_{1}$ are constants.

Theorem 7.2. Let $\gamma$ be a unit speed spacelike curve in $E_{1}^{3}$ with Darboux vector $\vec{W}$. Then,

$$
\Delta \vec{W}+\lambda \nabla_{\gamma^{\prime}} \vec{W}+\mu \vec{W}=0,
$$

holds along the curve $\gamma$ for the constants $\lambda$ and $\mu$ if and only if $\gamma$ is a general spacelike helix, with curvature and the torsion

$$
\begin{gathered}
\kappa=c \tau, \\
\tau=c_{1} \exp \left(\frac{-\lambda+\sqrt{\lambda^{2}+4 \mu}}{2} s\right)+c_{2} \exp \left(\frac{-\lambda-\sqrt{\lambda^{2}+4 \mu}}{2} s\right),
\end{gathered}
$$

respectively, where $c, c_{1}, c_{2}$ are constants.

Theorem 7.3. Let $\gamma$ be a unit speed spacelike curve in $E_{1}^{3}$. Then, $\vec{W}^{\perp}$ is harmonic 1-type if and only if

$$
\left.\kappa^{\prime \prime 2}\right) \kappa=0, \quad 2 \kappa^{\prime} \tau+\tau^{\prime} \kappa=0 .
$$

Corollary 8. Let $\gamma$ be a unit speed spacelike curve in $E_{1}^{3}$ with Darboux vector $\vec{W}$. If $\gamma$ is a circular spacelike helix with torsion $\lambda=-\tau^{2}$, then the normal component $\vec{W}^{\perp}$ of the Darboux vector field is of harmonic 1-type.

\section{Conclusions.}

In the space, while the position vector drawing the space curve, the Frenet frame of the curve makes a rotation around an axis which is called Darboux instantaneous rotation vector. In this study, we give some characterizations on the Darboux instantaneous rotation vector field of the curves in Minkowski 3-space $E_{1}^{3}$ by using Laplacian and normal Laplacian operators. We define harmonic type and harmonic 
1-type Darboux vector and show that the curves having harmonic type and harmonic 1-type Darboux vectors are general helices in Minkowski 3-space.

\section{REFERENCES}

[1] A. Altın, Harmonic curvatures of non-null curves and the helix in $R_{v}^{n}$, Hacettepe Bul. of Nat. Sci. and Eng., Vol. 30 (2001) 55-61.

[2] K. Arslan, Y. Aydın, G. Öztürk, H. Ugail, Biminimal Curves in Euclidean Spaces, International Electronic Journal of Geometry, 2 (2009) 46-52.

[3] M. Barros, O.J. Gray, On Submanifolds with Harmonic Mean Curvature, Proc. Amer. Math. Soc., 123 (1995) 2545-2549.

[4] B.Y. Chen, S. Ishikawa, Biharmonic surface in pseudo-Euclidean spaces, Mem. Fac. Sci. Kyushu Univ., A 45 (1991) 323-347.

[5] N. Chouaieb, A. Goriely, J.H. Maddocks, Helices, PNAS 103 (25) (2006) 9398-9403.

[6] A. Ferrandez, P. Lucas, M.A. Merono, Biharmonic Hopf cylinders, Rocky Mountain J., 28 (1998) 957-975.

[7] H.H. Hacısalihoğlu, R. Öztürk, On the characterization of inclined curves in $E^{n}$ - I., Tensor, N., S., 64 (2003) 157-162.

[8] H.H. Hacısalihoğlu, R. Öztürk, On the characterization of inclined curves in $E^{n}$ - II., Tensor, N., S., 64 (2003) 163-169.

[9] S. Izumiya, N. Takeuchi, New special curves and developable surfaces, Turk J. Math. Vol. 28 (2004) 153-163.

[10] B. Kılıç, K. Arslan, Harmonic 1-type submanifolds of Euclidean spaces, Int. J. Math. Stat., 3 (2008) A08, 47-53.

[11] H. Kocayiğit, Biharmonic Curves in Lorentz 3-Manifolds and Contact Geometry, Ph. D. Thesis, Ankara University, (2004).

[12] H. Kocayiğit, M. Önder, Timelike curves of constant slope in Minkowski space $E_{1}^{4}$, BU/JST, Vol. 1 (2) (2007) 311-318.

[13] A. Lucas Amand, P. Lambin, Diffraction by DNA, carbon nanotubes and other helical nanostructures, Rep. Prog. Phys. 68 (2005) 1181-1249.

[14] A. Mağden, On the curves of constant slope, YYÜ Fen Bilimleri Dergisi, Vol. 4 (1993) 103-109.

[15] B. O'neill, Semi-Riemannian Geometry, Academic Press 1983.

[16] M. Petrovic-Torgasev, E. Sucurovic, W-curves in Minkowski spac-time, Novi Sad J. Math., Vol. 32 No. 2 (2002) 55-65.

[17] D.J. Struik, Lectures on Classical Differential Geometry, 2nd ed. Addison Wesley, Dover, (1988).

[18] H.H. Uğurlu, A. Çalışkan, Darboux Ani Dönme Vektörleri ile Spacelike ve Timelike Yüzeyler Geometrisi, Celal Bayar Üniversitesi Yayınları, Yayın No: 0006. (2012).

[19] J. Walrave, Curves and surfaces in Minkowski space, Doctoral thesis, K. U. Leuven, Fac. Of Science, Leuven, (1995).

[20] J.D. Watson, F.H.C. Crick, Genetic implications of the structure of deoxyribonucleic acid, Nature, 171 (1953) 964-967.

[21] X. Yang, High accuracy approximation of helices by quintic curve, Comput. Aided Geometric Design, 20 (2003) 303-317.

Current address: Hüseyin Kocayiğit and Mehmet Önder; Department of Mathematics Faculty of Science and Arts Celal Bayar University, 45047, Manisa, TURKEY

Kadri Arslan; Department of Mathematics Science and Arts Faculty Uludağ University, 16059

Bursa, TURKEY

E-mail address: huseyin.kocayigit@bayar.edu.tr, mehmet.onder@bayar.edu.tr, mehmetlider@mynet.com

$U R L:$ http://communications.science.ankara.edu.tr/index.php?series=A1 